\title{
A new class of degenerate Hermite poly-Genocchi polynomials
}

\section{Waseem A. Khan ${ }^{1}$ and Nisar $\mathbf{K ~ S}^{2}$}

${ }^{1}$ Department of Mathematics, Faculty of Science, Integral University, Lucknow-226026, India

${ }^{2}$ Department of Mathematics, College of Arts and Science-Wadi Al dawaser, Prince Sattam bin Abdulaziz University, Riyadh region 11991, Saudi Arabia

E-mail: waseem08_khan@rediffmail.com, n.sooppy@psau.edu.sa

Abstract. In this article, authors introduce a new class of degenerate Hermite polyGenocchi polynomials and give some identities of these polynomials related to the Stirling numbers of the second kind. Some implicit summation formulae and general symmetry identities are derived by using different analytical means and applying generating functions. These results extend some known summations and identities of degenerate Hermite poly-Bernoulli numbers and polynomials studied by Khan [8].

Keywords: Hermite polynomials, degenerate Hermite poly-Genocchi polynomials, summation formulae, symmetric identities.

2010 Mathematics Subject Classification.: 05A10, 05A15, 33C45, 33C99.

\section{Introduction}

The 2-variable Hermite-Kampé de Fériet polynomials $(2 \mathrm{VHKdFP}) H_{n}(x, y)[3$, 5] are defined as

$$
H_{n}(x, y)=n ! \sum_{r=0}^{\left[\frac{n}{2}\right]} \frac{y^{r} x^{n-2 r}}{r !(n-2 r) !}
$$

From (1.1) is

$$
e^{x t+y t^{2}}=\sum_{n=0}^{\infty} H_{n}(x, y) \frac{t^{n}}{n !}
$$

When $y=-1$ and $x$ is replaced by $2 x$ in (1.2), the result reduces to ordinary Hermite polynomials $H_{n}(x)$ (see [1]).

The generating function for degenerate Hermite polynomials are given by

$$
(1+\lambda t)^{\frac{x}{\lambda}}\left(1+\lambda t^{2}\right)^{\frac{y}{\lambda}}=\sum_{n=0}^{\infty} H_{n}(x, y ; \lambda) \frac{t^{n}}{n !}, \quad(\text { see }[8]) .
$$

where $\lambda \neq 0$. Since $(1+\lambda t)^{\frac{1}{\lambda}} \longrightarrow e^{t}$ as $\lambda \longrightarrow 0$, it is evident that (1.3) reduces to (1.2). That is $H_{n}(x, y)$ limiting case of $H_{n}(x, y ; \lambda)$ when $\lambda \longrightarrow 0$.

By equating coefficients of $t^{n}$ on both the sides of (1.3), the following representation of $H_{n}(x, y ; \lambda)$ is obtained

$$
H_{n}(x, y ; \lambda)=n ! \sum_{r=0}^{\left[\frac{n}{2}\right]} \frac{\left(-\frac{x}{\lambda}\right)_{n-2 r}\left(-\frac{y}{\lambda}\right)_{r}(-\lambda)^{n-r}}{r !(n-2 r) !} .
$$

Since $\lim _{\lambda \longrightarrow 0} H_{n}(x, y ; \lambda)=H_{n}(x, y),(1.1)$ is a limiting case of (1.4).

The classical Genocchi numbers $G_{n}$, the calssical Genocchi polynomials $G_{n}(x)$ and the generalized Genocchi polynomilas $G_{n}^{(\alpha)}(x)$ of (real or complex) order $\alpha$ are 
usually defined by means of the following generating functions (see for details[1], see also [8-14] and the references cited therein):

$$
\begin{gathered}
\left(\frac{2 t}{e^{t}+1}\right)=\sum_{n=0}^{\infty} G_{n} \frac{t^{n}}{n !}(|t|<\pi), \\
\left(\frac{2 t}{e^{t}+1}\right)^{\alpha} e^{x t}=\sum_{n=0}^{\infty} G_{n}^{(\alpha)} \frac{t^{n}}{n !}(|t|<\pi),
\end{gathered}
$$

and

$$
\left(\frac{2 t}{e^{t}+1}\right)^{\alpha} e^{x t}=\sum_{n=0}^{\infty} G_{n}^{(\alpha)} \frac{t^{n}}{n !}\left(|t|<\pi ; 1^{\alpha}=1\right),
$$

with

respectively.

$$
G_{n}^{1}(0)=G_{n}(0)=G_{n}
$$

For $\lambda \in \mathbb{C}$, Carlitz [4] introduced the degenerate Bernoulli polynomials defined by

$$
\frac{t}{(1+\lambda t)^{\frac{1}{\lambda}}-1}(1+\lambda t)^{\frac{x}{\lambda}}=\sum_{n=0}^{\infty} \beta_{n}(x ; \lambda) \frac{t^{n}}{n !}
$$

so that

$$
\beta_{n}(x ; \lambda)=\sum_{n=0}^{m}\left(\begin{array}{l}
n \\
m
\end{array}\right) \beta_{m}(\lambda)\left(\frac{x}{\lambda}\right)_{n-m} .
$$

When $x=0, \beta_{n}(\lambda)=\beta_{n}(0 ; \lambda)$ are called the degenerate Bernoulli numbers.

From (1.10), we note that

$$
\begin{gathered}
\sum_{n=0}^{\infty} \lim _{\lambda \longrightarrow 0} \beta_{n}(x ; \lambda) \frac{t^{n}}{n !}=\lim _{\lambda \longrightarrow 0} \frac{t}{(1+\lambda t)^{\frac{1}{\lambda}}-1}(1+\lambda t)^{\frac{x}{\lambda}} \\
=\frac{t}{e^{t}-1} e^{x t}=\sum_{n=0}^{\infty} B_{n}(x) \frac{t^{n}}{n !} .
\end{gathered}
$$

where $B_{n}(x)$ are called the Bernoulli polynomials (see [7-14]).

The classical polylogarithm function $\operatorname{Li}_{k}(z)$ is

$$
\operatorname{Li}_{k}(z)=\sum_{m=1}^{\infty} \frac{z^{m}}{m^{k}},(k \in \mathbb{Z}) \quad(\text { see }[6,10-13])
$$

so for $k \leq 1$,

$$
\operatorname{Li}_{k}(z)=-\ln (1-z), \operatorname{Li}_{0}(z)=\frac{z}{1-z}, \operatorname{Li}_{-1}(z)=\frac{z}{(1-z)^{2}}, \ldots
$$

The poly-Bernoulli polynomials are given by

$$
\frac{\operatorname{Li}_{k}\left(1-e^{-t}\right)}{e^{t}-1} e^{x t}=\sum_{n=0}^{\infty} B_{n}^{(k)}(x) \frac{t^{n}}{n !}, \quad(\text { see }[2,10,13])
$$

For $k=1$ in (1.13), we have

$$
\frac{\mathrm{Li}_{1}\left(1-e^{-t}\right)}{e^{t}-1} e^{x t}=\frac{t}{e^{t}-1} e^{x t}=\sum_{n=0}^{\infty} B_{n}(x) \frac{t^{n}}{n !} .
$$

From (1.13) and (1.14), we have

$$
B_{n}^{(1)}(x)=B_{n}(x)
$$


Very recently, Khan [8] introduced the degenerate Hermite poly-Bernoulli polynomials of two variables $\beta_{n}^{(\alpha)}(x, y ; \lambda)$ defined by

$$
\frac{\operatorname{Li}_{k}\left(1-e^{-t}\right)}{(1+\lambda t)^{\frac{1}{\lambda}}-1}(1+\lambda t)^{\frac{x}{\lambda}}\left(1+\lambda t^{2}\right)^{\frac{y}{\lambda}}=\sum_{n=0}^{\infty} H \beta_{n}^{(k)}(x, y ; \lambda) \frac{t^{n}}{n !} .
$$

Note that

$$
\lim _{\lambda \longrightarrow 0} \frac{\operatorname{Li}_{k}\left(1-e^{-t}\right)}{(1+\lambda t)^{\frac{1}{\lambda}}-1}(1+\lambda t)^{\frac{x}{\lambda}}\left(1+\lambda t^{2}\right)^{\frac{y}{\lambda}}=\sum_{n=0}^{\infty}{ }_{H} B_{n}^{(k)}(x, y) \frac{t^{n}}{n !} .
$$

For $k=1$ in (1.16), the result reduces to known result of Dattoli et al. [5]. We recall the following definition as follows:

The Stirling number of the first kind is given by

$$
(x)_{n}=x(x-1) \cdots(x-n+1)=\sum_{l=0}^{n} S_{1}(n, l) x^{l},(n \geq 0) .
$$

and the Stirling number of the second kind is defined by generating function to be

$$
\left(e^{t}-1\right)^{n}=n ! \sum_{l=n}^{\infty} S_{2}(l, n) \frac{t^{l}}{l !} .
$$

A generalized falling factorial sum $\tau_{k}(n ; \lambda)$ can be defined by the generating function [14]:

$$
\sum_{k=0}^{\infty} \tau_{k}(n ; \lambda) \frac{t^{k}}{k !}=\frac{1-(-(1+\lambda t))^{\frac{(n+1)}{\lambda}}}{1+(1+\lambda t)^{\frac{1}{\lambda}}} .
$$

where $\lim _{\lambda \longrightarrow 0} \tau_{k}(n ; \lambda)=T_{k}(n)$.

In this paper, we consider a new class of degenerate Hermite poly-Genocchi polynomials ${ }_{H} G_{n, \lambda}^{(k)}(x, y)$ and develop some elementary properties and derive some implicit formulae and symmetric identities for the degenerate Hermite poly-Genocchi polynomials by using different analytical means of their respective generating functions.

\section{A new class of degenerate Hermite poly-Genocchi polynomials}

For $\lambda \in \mathbb{C}, k \in \mathbb{Z}$, we consider the degenerate Hermite poly-Genocchi polynomials which are given by the generating function

$$
\frac{2 \operatorname{Li}_{k}\left(1-e^{-t}\right)}{(1+\lambda t)^{\frac{1}{\lambda}}+1}(1+\lambda t)^{\frac{x}{\lambda}}\left(1+\lambda t^{2}\right)^{\frac{y}{\lambda}}=\sum_{n=0}^{\infty}{ }_{H} G_{n, \lambda}^{(k)}(x, y) \frac{t^{n}}{n !},
$$

so that

$$
{ }_{H} G_{n, \lambda}^{(k)}(x, y)=\sum_{m=0}^{n}\left(\begin{array}{c}
n \\
m
\end{array}\right) G_{m, \lambda}^{(k)} H_{n-m}(x, y ; \lambda) .
$$

When $x=y=0$ in $(2.1), G_{n, \lambda}^{(k)}={ }_{H} G_{n, \lambda}^{(k)}(0,0)$ are called the degenerate poly-Genocchi numbers. Note that

and

$$
{ }_{H} G_{n, \lambda}^{(1)}(x, y)={ }_{H} G_{n, \lambda}(x, y)
$$

$$
\lim _{\lambda \longrightarrow 0}{ }_{H} G_{n, \lambda}^{(k)}(x, y)={ }_{H} G_{n}^{(k)}(x, y),(k \in \mathbb{Z}) .
$$

where ${ }_{H} G_{n}^{(k)}(x, y)$ are called the Hermite poly-Genocchi polynomials (see[12] ). 
For $y=0$ in (2.1), we have

$$
\frac{2 \operatorname{Li}_{k}\left(1-e^{-t}\right)}{(1+\lambda t)^{\frac{1}{\lambda}}+1}(1+\lambda t)^{\frac{x}{\lambda}}=\sum_{n=0}^{\infty} G_{n, \lambda}^{(k)}(x) \frac{t^{n}}{n !},(\lambda \in \mathbb{C}, k \in \mathbb{Z}) .
$$

Theorem 2.1. For $n \geq 0$, we have

$$
{ }_{H} G_{n, \lambda}^{(2)}(x, y)=\sum_{m=0}^{n}\left(\begin{array}{c}
n \\
m
\end{array}\right) \frac{B_{m} m !}{m+1}{ }_{H} G_{n-m, \lambda}(x, y) .
$$

Proof. Applying Definition (2.1), we have

$$
\begin{gathered}
\sum_{n=0}^{\infty}{ }_{H} G_{n}^{(k)}(x, y ; \lambda) \frac{t^{n}}{n !}=\frac{2 \operatorname{Li}_{k}\left(1-e^{-t}\right)}{(1+\lambda t)^{\frac{1}{\lambda}}+1}(1+\lambda t)^{\frac{x}{\lambda}}\left(1+\lambda t^{2}\right)^{\frac{y}{\lambda}} \\
=\frac{2(1+\lambda t)^{\frac{x}{\lambda}}\left(1+\lambda t^{2}\right)^{\frac{y}{\lambda}}}{(1+\lambda t)^{\frac{1}{\lambda}}+1} \int_{0}^{t} \underbrace{\frac{1}{e^{z}-1} \int_{0}^{t} \frac{1}{e^{z}-1} \cdots \frac{1}{e^{z}-1} \int_{0}^{t} \frac{z}{e^{z}-1}}_{(k-2)-\text { times }} d z \cdots d z
\end{gathered}
$$

For $k=2$ in (2.6), we have

$$
\begin{gathered}
\sum_{n=0}^{\infty}{ }_{H} G_{n, \lambda}^{(2)}(x, y) \frac{t^{n}}{n !}=\frac{2(1+\lambda t)^{\frac{x}{\lambda}}\left(1+\lambda t^{2}\right)^{\frac{y}{\lambda}}}{(1+\lambda t)^{\frac{1}{\lambda}}+1} \int_{0}^{t} \frac{z}{e^{z}-1} d z \\
=\left(\sum_{m=0}^{\infty} \frac{B_{m} t^{m}}{m+1}\right) \frac{2(1+\lambda t)^{\frac{x}{\lambda}}\left(1+\lambda t^{2}\right)^{\frac{y}{\lambda}}}{(1+\lambda t)^{\frac{1}{\lambda}}+1} \\
=\left(\sum_{m=0}^{\infty} \frac{B_{m} m !}{m+1} \frac{t^{m}}{m !}\right)\left(\sum_{n=0}^{\infty} H_{n, \lambda}(x, y) \frac{t^{n}}{n !}\right)
\end{gathered}
$$

Replacing $n$ by $n-m$ in above equation, we have

$$
=\sum_{n=0}^{\infty}\left(\sum_{m=0}^{n}\left(\begin{array}{l}
n \\
m
\end{array}\right) \frac{B_{m} m !}{m+1}{ }_{H} G_{n-m, \lambda}(x, y)\right) \frac{t^{n}}{n !}
$$

On equating the coefficients of the like powers of $\frac{t^{n}}{n !}$ in the above equation, we get the result (2.5).

Theorem 2.2. For $n \geq 0$, we have

$$
{ }_{H} G_{n, \lambda}^{(k)}(x, y)=\sum_{p=0}^{n}\left(\begin{array}{c}
n \\
p
\end{array}\right)\left(\sum_{l=1}^{p+1} \frac{(-1)^{l+p+1} l ! S_{2}(p+1, l)}{l^{k}(p+1)}\right){ }_{H} G_{n-p, \lambda}(x, y) .
$$

Proof. From equation (2.1), we have

$$
\sum_{n=0}^{\infty}{ }_{H} G_{n, \lambda}^{(k)}(x, y) \frac{t^{n}}{n !}=\left(\frac{\operatorname{Li}_{k}\left(1-e^{-t}\right)}{t}\right)\left(\frac{2 t(1+\lambda t)^{\frac{x}{\lambda}}\left(1+\lambda t^{2}\right)^{\frac{y}{\lambda}}}{(1+\lambda t)^{\frac{1}{\lambda}}+1}\right)
$$

Now

$$
\begin{aligned}
\frac{1}{t} \operatorname{Li}_{k}\left(1-e^{-t}\right) & =\frac{1}{t} \sum_{l=1}^{\infty} \frac{\left(1-e^{-t}\right)^{l}}{l^{k}}=\frac{1}{t} \sum_{l=1}^{\infty} \frac{(-1)^{l}}{l^{k}}\left(1-e^{-t}\right)^{l} \\
= & \frac{1}{t} \sum_{l=1}^{\infty} \frac{(-1)^{l}}{l^{k}} l ! \sum_{p=l}^{\infty}(-1)^{p} S_{2}(p, l) \frac{t^{p}}{p !} \\
= & \frac{1}{t} \sum_{p=1}^{\infty} \sum_{l=1}^{p} \frac{(-1)^{l+p}}{l^{k}} l ! S_{2}(p, l) \frac{t^{p}}{p !}
\end{aligned}
$$




$$
=\sum_{p=0}^{\infty}\left(\sum_{l=1}^{p+1} \frac{(-1)^{l+p+1}}{l^{k}} l ! \frac{S_{2}(p+1, l)}{p+1}\right) \frac{t^{p}}{p !}
$$

From equations (2.8) and (2.9), we have

$$
\sum_{n=0}^{\infty}{ }_{H} G_{n, \lambda}^{(k)}(x, y) \frac{t^{n}}{n !}=\sum_{p=0}^{\infty}\left(\sum_{l=1}^{p+1} \frac{(-1)^{l+p+1}}{l^{k}} l ! \frac{S_{2}(p+1, l)}{p+1}\right) \frac{t^{p}}{p !}\left(\sum_{n=0}^{\infty}{ }_{H} G_{n, \lambda}(x, y) \frac{t^{n}}{n !}\right)
$$

Replacing $n$ by $n-p$ in the r.h.s of above equation and comparing the coefficients of $\frac{t^{n}}{n !}$, we get the result $(2.7)$.

Theorem 2.3. For $n \geq 1$, we have

$$
\begin{gathered}
{ }_{H} G_{n, \lambda}^{(k)}(x+1, y)+{ }_{H} G_{n, \lambda}^{(k)}(x, y) \\
=2 \sum_{p=1}^{n}\left(\begin{array}{c}
n \\
p
\end{array}\right)\left(\sum_{l=0}^{p-1} \frac{(-1)^{l+p+1}}{(l+1)^{k}}(l+1) ! S_{2}(p, l+1)\right) H_{n-p}(x, y ; \lambda) .
\end{gathered}
$$

Proof. Using the definition (2.1), we have

$$
\begin{gathered}
\sum_{n=0}^{\infty}{ }_{H} G_{n, \lambda}^{(k)}(x+1, y) \frac{t^{n}}{n !}+\sum_{n=0}^{\infty}{ }_{H} G_{n, \lambda}^{(k)}(x, y) \frac{t^{n}}{n !} \\
=\frac{2 \operatorname{Li}_{k}\left(1-e^{-t}\right)}{(1+\lambda t)^{\frac{1}{\lambda}}+1}(1+\lambda t)^{\frac{x+1}{\lambda}}\left(1+\lambda t^{2}\right)^{\frac{y}{\lambda}}+\frac{2 \operatorname{Li}_{k}\left(1-e^{-t}\right)}{(1+\lambda t)^{\frac{1}{\lambda}}+1}(1+\lambda t)^{\frac{x}{\lambda}}\left(1+\lambda t^{2}\right)^{\frac{y}{\lambda}} \\
=2 \operatorname{Li}_{k}\left(1-e^{-t}\right)(1+\lambda t)^{\frac{x}{\lambda}}\left(1+\lambda t^{2}\right)^{\frac{y}{\lambda}} \\
=2 \sum_{l=0}^{\infty} \frac{\left(1-e^{-t}\right)^{l+1}}{(l+1)^{k}}(1+\lambda t)^{\frac{x}{\lambda}}\left(1+\lambda t^{2}\right)^{\frac{y}{\lambda}} \\
=2 \sum_{p=1}^{\infty}\left(\sum_{l=0}^{p-1} \frac{(-1)^{l+p+1}}{(l+1)^{k}}(l+1) ! S_{2}(p, l+1)\right) \frac{t^{p}}{p !}(1+\lambda t)^{\frac{x}{\lambda}}\left(1+\lambda t^{2}\right)^{\frac{y}{\lambda}} \\
=2\left(\sum_{p=1}^{\infty}\left(\sum_{l=0}^{p-1} \frac{(-1)^{l+p+1}}{(l+1)^{k}}(l+1) ! S_{2}(p, l+1)\right) \frac{t^{p}}{p !}\right)\left(\sum_{n=0}^{\infty} H_{n}(x, y ; \lambda) \frac{t^{n}}{n !}\right)
\end{gathered}
$$

Replacing $n$ by $n-p$ in the above equation and comparing the coefficients of $\frac{t^{n}}{n !}$, we get the result $(2.10)$.

Theorem 2.4. For $n \geq 0, d \in \mathbb{N}$ and $k \in \mathbb{Z}$, we have

${ }_{H} G_{n, \lambda}^{(k)}(x, y)=\sum_{a=0}^{d-1} \sum_{l=0}^{n} \sum_{p=1}^{l+1}\left(\begin{array}{c}n \\ l\end{array}\right) d^{n-l-1} \frac{(-1)^{l+p+1} p ! S_{2}(l+1, p)}{p^{k} l+1}{ }_{H} G_{n-l}\left(\frac{l+x}{d}, y ; \frac{\lambda}{d}\right)$.

Proof. From equation (2.1), we can be written as

$$
\begin{gathered}
\sum_{n=0}^{\infty}{ }_{H} G_{n, \lambda}^{(k)}(x, y) \frac{t^{n}}{n !}=\frac{2 \operatorname{Li}_{k}\left(1-e^{-t}\right)}{(1+\lambda t)^{\frac{1}{\lambda}}+1}(1+\lambda t)^{\frac{x}{\lambda}}\left(1+\lambda t^{2}\right)^{\frac{y}{\lambda}} \\
=\frac{2 \operatorname{Li}_{k}\left(1-e^{-t}\right)}{(1+\lambda t)^{\frac{d}{\lambda}}+1} \sum_{a=0}^{d-1}(1+\lambda t)^{\frac{l+x}{\lambda}}\left(1+\lambda t^{2}\right)^{\frac{y}{\lambda}} \\
=\left(\frac{\operatorname{Li}_{k}\left(1-e^{-t}\right)}{t}\right) \frac{1}{d} \sum_{a=0}^{d-1} \frac{2 d t}{(1+\lambda t)^{\frac{d}{\lambda}}+1}(1+\lambda t)^{\frac{l+x}{\lambda}}\left(1+\lambda t^{2}\right)^{\frac{y}{\lambda}}
\end{gathered}
$$




$$
=\left(\sum_{l=0}^{\infty}\left(\sum_{p=1}^{l+1} \frac{(-1)^{l+p+1}}{p^{k}} p ! \frac{S_{2}(l+1, p)}{l+1}\right) \frac{t^{l}}{l !}\right)\left(\sum_{n=0}^{\infty} d^{n-1} \sum_{a=0}^{d-1}{ }_{H} G_{n}\left(\frac{l+x}{d}, y ; \frac{\lambda}{d}\right) \frac{t^{n}}{n !}\right) .
$$

Replacing $n$ by $n-l$ in above equation and comparing the coefficient of $\frac{t^{n}}{n !}$, we get the result (2.11).

\section{Implicit summation formulae involving degenerate Hermite poly-Genocchi polynomials}

In this section, we establish some implicit summation formulae for degenerate Hermite poly-Genocchi polynomials ${ }_{H} G_{n, \lambda}^{(k)}(x, y)$ as follows.

Theorem 3.1. The following implicit summation formula involving degenerate Hermite poly-Genocchi polynomials ${ }_{H} G_{n, \lambda}^{(k)}(x, y)$ holds true:

$$
{ }_{H} G_{n, \lambda}^{(k)}(x+u, y+w)=\sum_{m=0}^{n}\left(\begin{array}{l}
n \\
m
\end{array}\right){ }_{H} G_{n-m}^{(k)}(x, y) H_{m}(u, w ; \lambda) .
$$

Proof. By the definition of degenerate poly-Genocchi polynomials and the definition (1.3), we have

$$
\frac{2 \operatorname{Li}_{k}\left(1-e^{-t}\right)}{(1+\lambda t)^{\frac{1}{\lambda}}+1}(1+\lambda t)^{\frac{x+u}{\lambda}}\left(1+\lambda t^{2}\right)^{\frac{y+w}{\lambda}}=\left(\sum_{n=0}^{\infty}{ }_{H} G_{n, \lambda}^{(k)}(x, y) \frac{t^{n}}{n !}\right)\left(\sum_{m=0}^{\infty} H_{m}(u, w ; \lambda) \frac{t^{m}}{m !}\right)
$$

Replacing $n$ by $n-m$ in above equation and comparing the coefficients of $\frac{t^{n}}{n !}$, we get the result (3.1).

Theorem 3.2. The following implicit summation formula involving degenerate Hermite poly-Genocchi polynomials ${ }_{H} G_{n, \lambda}^{(k)}(x, y)$ holds true:

$$
{ }_{H} G_{n, \lambda}^{(k)}(x, y)=\sum_{m=0}^{n-2 j} \sum_{j=0}^{\left[\frac{n}{2}\right]} G_{m, \lambda}^{(k)}\left(-\frac{x}{\lambda}\right)_{n-m-2 j}(-\lambda)^{n-m-j}\left(-\frac{y}{\lambda}\right)_{j} \frac{n !}{m ! j !(n-2 j-m) !} .
$$

Proof. Applying the definition $(2.1)$ to the term $\frac{2 \operatorname{Li}_{k}\left(1-e^{-t}\right)}{(1+\lambda t)^{\frac{1}{\lambda}}+1}$ and expanding the function $(1+\lambda t)^{\frac{x}{\lambda}}\left(1+\lambda t^{2}\right)^{\frac{y}{\lambda}}$ at $t=0$ yields

$$
\begin{gathered}
\frac{2 \operatorname{Li}_{k}\left(1-e^{-t}\right)}{(1+\lambda t)^{\frac{1}{\lambda}}+1}(1+\lambda t)^{\frac{x}{\lambda}}\left(1+\lambda t^{2}\right)^{\frac{y}{\lambda}}=\left(\sum_{m=0}^{\infty} G_{m, \lambda}^{(k)} \frac{t^{m}}{m !}\right)\left(\sum_{n=0}^{\infty}\left(-\frac{x}{\lambda}\right)_{n} \frac{(-\lambda t)^{n}}{n !}\right)\left(\sum_{j=0}^{\infty}\left(-\frac{y}{\lambda}\right)_{j} \frac{\left(-\lambda t^{2}\right)^{j}}{j !}\right) \\
=\sum_{n=0}^{\infty}\left(\sum_{m=0}^{n}\left(\begin{array}{c}
n \\
m
\end{array}\right) G_{m, \lambda}^{(k)}\left(-\frac{x}{\lambda}\right)_{n-m}(-\lambda)^{n-m}\right) \frac{t^{n}}{n !}\left(\sum_{j=0}^{\infty}\left(-\frac{y}{\lambda}\right)_{j} \frac{\left(-\lambda t^{2}\right)^{j}}{j !}\right)
\end{gathered}
$$

Replacing $n$ by $n-2 j$, we have

$$
\begin{gathered}
\sum_{n=0}^{\infty}{ }_{H} G_{n, \lambda}^{(k)}(x, y) \frac{t^{n}}{n !} \\
=\sum_{n=0}^{\infty}\left(\sum_{m=0}^{n-2 j} \sum_{j=0}^{\left[\frac{n}{2}\right]}\left(\begin{array}{l}
n-2 j \\
m
\end{array}\right) G_{m, \lambda}^{(k)}\left(-\frac{x}{\lambda}\right)_{n-m-2 j}(-\lambda)^{n-m-j}\left(-\frac{y}{\lambda}\right)_{j}\right) \frac{t^{n}}{(n-2 j) ! j !} .
\end{gathered}
$$


Equating their coefficients of $\frac{t^{n}}{n !}$, we get the result (3.2).

Theorem 3.3. The following implicit summation formula involving degenerate Hermite poly-Genocchi polynomials ${ }_{H} G_{n, \lambda}^{(k)}(x, y)$ holds true:

$$
{ }_{H} G_{n, \lambda}^{(k)}(x, y)=\sum_{m=0}^{n}\left(\begin{array}{c}
n \\
m
\end{array}\right)\left(-\frac{z}{\lambda}\right)_{n-m}(-\lambda)^{n-m}{ }_{H} G_{m, \lambda}^{(k)}(x-z, y) .
$$

Proof. By exploiting the generating function (2.1), we can write the equation

$$
\begin{gathered}
\sum_{n=0}^{\infty}{ }_{H} G_{n, \lambda}^{(k)}(x, y) \frac{t^{n}}{n !}=\frac{2 \operatorname{Li}_{k}\left(1-e^{-t}\right)}{(1+\lambda t)^{\frac{1}{\lambda}}+1}(1+\lambda t)^{\frac{x-z}{\lambda}}\left(1+\lambda t^{2}\right)^{\frac{y}{\lambda}}(1+\lambda t)^{\frac{z}{\lambda}} \\
=\left(\sum_{m=0}^{\infty} H_{m, \lambda}^{(k)}(x-z, y) \frac{t^{m}}{m !}\right)\left(\sum_{n=0}^{\infty}\left(-\frac{z}{\lambda}\right)_{n} \frac{(-\lambda t)^{n}}{n !}\right)
\end{gathered}
$$

Replacing $n$ by $n-m$ in above equation and equating their coefficients of $\frac{t^{n}}{n !}$ leads to formula (3.4).

Theorem 3.4. The following implicit summation formula involving degenerate Hermite poly-Genocchi polynomials ${ }_{H} G_{n, \lambda}^{(k)}(x, y)$ holds true:

$$
{ }_{H} G_{n, \lambda}^{(k)}(x+1, y)=\sum_{r=0}^{n}\left(\begin{array}{c}
n \\
r
\end{array}\right)\left(-\frac{1}{\lambda}\right)_{r}(-\lambda)^{r}{ }_{H} G_{n-r, \lambda}^{(k)}(x, y) .
$$

Proof. By the definition of degenerate Hermite poly-Genocchi polynomials, we have

$$
\begin{aligned}
\sum_{n=0}^{\infty}{ }_{H} G_{n, \lambda}^{(k)}(x+1, y) \frac{t^{n}}{n !}-\sum_{n=0}^{\infty}{ }_{H} G_{n, \lambda}^{(k)}(x, y) \frac{t^{n}}{n !}=\frac{2 \operatorname{Li}_{k}\left(1-e^{-t}\right)}{(1+\lambda t)^{\frac{1}{\lambda}}+1}(1+\lambda t)^{\frac{x}{\lambda}}\left(1+\lambda t^{2}\right)^{\frac{y}{\lambda}}\left((1+\lambda t)^{\frac{1}{\lambda}}-1\right) \\
=\left(\sum_{n=0}^{\infty}{ }_{H} G_{n, \lambda}^{(k)}(x, y) \frac{t^{n}}{n !}\right)\left(\sum_{r=0}^{\infty}\left(-\frac{1}{\lambda}\right)_{r} \frac{(-\lambda t)^{r}}{r !}\right)-\sum_{n=0}^{\infty}{ }_{H} G_{n, \lambda}^{(k)}(x, y) \frac{t^{n}}{n !} \\
=\sum_{n=0}^{\infty} \sum_{r=0}^{n}{ }_{H} G_{n-r, \lambda}^{(k)}(x, y)\left(-\frac{1}{\lambda}\right)_{r}(-\lambda)^{r} \frac{t^{n}}{(n-r) ! r !}-\sum_{n=0}^{\infty}{ }_{H} G_{n, \lambda}^{(k)}(x, y) \frac{t^{n}}{n !} .
\end{aligned}
$$

Finally, equating the coefficients of the like powers of $\frac{t^{n}}{n !}$, we get (3.6).

\section{General symmetry identities for degenerate Hermite poly-Genocchi polynomials}

In this section, we give general symmetry identities for the degenerate polyGenocchi polynomials $G_{n, \lambda}^{(k)}(x)$ and the degenerate Hermite poly-Genocchi polynomials ${ }_{H} G_{n, \lambda}^{(k)}(x, y)$ by applying the generating function(2.1) and (2.4).

Theorem 4.1. Let $a, b>0$ and $a \neq b$. For $x, y \in \mathbb{R}$ and $n \geq 0$, the following identity holds true:

$$
\begin{aligned}
& \sum_{m=0}^{n}\left(\begin{array}{c}
n \\
m
\end{array}\right) b^{m} a^{n-m}{ }_{H} G_{n-m, \lambda}^{(k)}\left(b x, b^{2} y\right)_{H} G_{m, \lambda}^{(k)}\left(a x, a^{2} y\right) \\
= & \sum_{m=0}^{n}\left(\begin{array}{c}
n \\
m
\end{array}\right) a^{m} b^{n-m}{ }_{H} G_{n-m, \lambda}^{(k)}\left(a x, a^{2} y\right)_{H} G_{m, \lambda}^{(k)}\left(b x, b^{2} y\right) .
\end{aligned}
$$


Proof. Let

$$
g(t)=\left(\frac{2 \operatorname{Li}_{k}\left(1-e^{-a t}\right) 2 \operatorname{Li}_{k}\left(1-e^{-b t}\right)}{\left((1+\lambda t)^{\frac{a}{\lambda}}+1\right)\left((1+\lambda t)^{\frac{b}{\lambda}}+1\right)}\right)(1+\lambda t)^{\frac{a b x}{\lambda}}\left(1+\lambda t^{2}\right)^{\frac{a^{2} b^{2} y}{\lambda}} .
$$

Then the expression for $g(t)$ is symmetric in $a$ and $b$ and we can expand $g(t)$ into series in two ways to obtain

$$
\begin{gathered}
g(t)=\sum_{n=0}^{\infty}{ }_{H} G_{n, \lambda}^{(k)}\left(b x, b^{2} y\right) \frac{(a t)^{n}}{n !} \sum_{m=0}^{\infty}{ }_{H} G_{m, \lambda}^{(k)}\left(a x, a^{2} y\right) \frac{(b t)^{m}}{m !} \\
=\sum_{n=0}^{\infty}\left(\sum_{m=0}^{n}\left(\begin{array}{c}
n \\
m
\end{array}\right) a^{n-m} b^{m}{ }_{H} G_{n-m, \lambda}^{(k)}\left(b x, b^{2} y\right)_{H} G_{m, \lambda}^{(k)}\left(a x, a^{2} y\right)\right) \frac{t^{n}}{n !} .
\end{gathered}
$$

Similarly, we can show that

$$
\begin{gathered}
g(t)=\sum_{n=0}^{\infty}{ }_{H} G_{n, \lambda}^{(k)}\left(a x, a^{2} y\right) \frac{(b t)^{n}}{n !} \sum_{m=0}^{\infty}{ }_{H} G_{m, \lambda}^{(k)}\left(b x, b^{2} y\right) \frac{(a t)^{m}}{m !} \\
=\sum_{n=0}^{\infty}\left(\sum_{m=0}^{n}\left(\begin{array}{c}
n \\
m
\end{array}\right) a^{m} b^{n-m}{ }_{H} G_{n-m, \lambda}^{(k)}\left(a x, a^{2} y\right)_{H} G_{m, \lambda}^{(k)}\left(b x, b^{2} y\right)\right) \frac{t^{n}}{n !} .
\end{gathered}
$$

Comparing the coefficients of $\frac{t^{n}}{n !}$ on the right hand sides of the last two equations, we arrive the desired result.

Remark 1. On setting $b=1$ in Theorem 4.1, we get the following result

$$
\begin{aligned}
& \sum_{m=0}^{n}\left(\begin{array}{c}
n \\
m
\end{array}\right) a^{n-m}{ }_{H} G_{n-m, \lambda}^{(k)}(x, y)_{H} G_{m, \lambda}^{(k)}\left(a x, a^{2} y\right) \\
= & \sum_{m=0}^{n}\left(\begin{array}{c}
n \\
m
\end{array}\right) a^{m}{ }_{H} G_{n-m, \lambda}^{(k)}\left(a x, a^{2} y\right)_{H} G_{m, \lambda}^{(k)}(x, y) .
\end{aligned}
$$

Theorem 4.2. For all integers $a>0, b>0$, and $n \geq 0$, the following identity holds true:

$$
\begin{aligned}
& \sum_{m=0}^{n}\left(\begin{array}{l}
n \\
m
\end{array}\right) a^{n-m} b_{H}^{m} G_{n-m, \lambda}^{(k)}\left(b x, b^{2} z\right) \sum_{i=0}^{m}\left(\begin{array}{l}
m \\
i
\end{array}\right) \tau_{i}(a-1 ; \lambda) G_{m-i, \lambda}^{(k)}(a y) \\
= & \sum_{m=0}^{n}\left(\begin{array}{l}
n \\
m
\end{array}\right) a^{m} b^{n-m}{ }_{H} G_{n-m, \lambda}^{(k)}\left(a x, a^{2} z\right) \sum_{i=0}^{m}\left(\begin{array}{l}
m \\
i
\end{array}\right) \tau_{i}(b-1 ; \lambda) G_{m-i, \lambda}^{(k)}(b y) .
\end{aligned}
$$

where generalized falling factorial sum $\tau_{k}(n ; \lambda)$ is given by $(1.19)$.

Proof. We now use

$$
g(t)=\frac{2 \operatorname{Li}_{k}\left(1-e^{-a t}\right) 2 \operatorname{Li}_{k}\left(1-e^{-b t}\right)\left(1-(-(1+\lambda t))^{\frac{a b}{\lambda}}\right)(1+\lambda t)^{\frac{a b(x+y)}{\lambda}}\left(1+\lambda t^{2}\right)^{\frac{a^{2} b^{2} z}{\lambda}}}{\left((1+\lambda t)^{\frac{a}{\lambda}}+1\right)\left((1+\lambda t)^{\frac{b}{\lambda}}+1\right)^{2}}
$$

to find that

$$
\begin{array}{r}
g(t)=\left(\frac{2 \operatorname{Li}_{k}\left(1-e^{-a t}\right)}{(1+\lambda t)^{\frac{a}{\lambda}}+1}\right)(1+\lambda t)^{\frac{a b x}{\lambda}}\left(1+\lambda t^{2}\right)^{\frac{a^{2} b^{2} z}{\lambda}}\left(\frac{1-(-(1+\lambda t))^{\frac{a b}{\lambda}}}{(1+\lambda t)^{\frac{b}{\lambda}}+1}\right) \\
\quad\left(\frac{2 \operatorname{Li}_{k}\left(1-e^{-b t}\right)}{(1+\lambda t)^{\frac{b}{\lambda}}+1}\right)(1+\lambda t)^{\frac{a b y}{\lambda}} \\
=\sum_{n=0}^{\infty}{ }_{H} G_{n, \lambda}^{(k)}\left(b x, b^{2} z\right) \frac{(a t)^{n}}{n !} \sum_{n=0}^{\infty} \tau_{n}(a-1 ; \lambda) \frac{(b t)^{n}}{n !} \sum_{n=0}^{\infty} G_{n, \lambda}^{(k)}(a y) \frac{(b t)^{n}}{n !}
\end{array}
$$




$$
\begin{gathered}
=\sum_{n=0}^{\infty}{ }_{H} G_{n, \lambda}^{(k)}\left(b x, b^{2} z\right) \frac{(a t)^{n}}{n !} \sum_{m=0}^{\infty} \sum_{i=0}^{m}\left(\begin{array}{l}
m \\
i
\end{array}\right) b^{m} \tau_{i}(a-1 ; \lambda) G_{m-i, \lambda}^{(k)}(a y) \frac{t^{m}}{m !} \\
=\sum_{n=0}^{\infty}\left(\sum_{m=0}^{n}\left(\begin{array}{l}
n \\
m
\end{array}\right) a^{n-m} b^{m}{ }_{H} G_{n-m, \lambda}^{(k)}\left(b x, b^{2} z\right) \sum_{i=0}^{m}\left(\begin{array}{l}
m \\
i
\end{array}\right) \tau_{i}(a-1 ; \lambda) G_{m-i, \lambda}^{(k)}(a y)\right) \frac{t^{n}}{n !} .
\end{gathered}
$$

By using a similar plan, we get

$$
g(t)=\sum_{n=0}^{\infty}\left(\sum_{m=0}^{n}\left(\begin{array}{l}
n \\
m
\end{array}\right) a^{m} b_{H}^{n-m} G_{n-m, \lambda}^{(k)}\left(a x, a^{2} z\right) \sum_{i=0}^{m}\left(\begin{array}{l}
m \\
i
\end{array}\right) \tau_{i}(b-1 ; \lambda) G_{m-i, \lambda}^{(k)}(b y)\right) \frac{t^{n}}{n !} .
$$

After comparing the coefficients of $\frac{t^{n}}{n !}$ on the right hand sides of the last two equations, we arrive at the desired result.

\section{References}

[1] Andrews, L. C, Special functions for engineers and mathematicians, Macmillan Co. New York, 1985.

[2] Bayad, A, Hamahata, Y, Multiple-Polylogarithms and multi-poly-Bernoulli polynomials, Funct. Approx. Comment. Math., 45(2012), 45-61.

[3] Bell, E. T, Exponential polynomials, Ann. of Math., 35(1934), 258-277.

[4] Carlitz, L, Degenerate Stirling Bernoulli and Eulerian numbers, Util. Math., 15(1979), 51-88.

[5] Dattoli, G, Lorenzutta, S and Cesarano, C, Finite sums and generalized forms of Bernoulli polynomials, Rendiconti di Mathematica, 19(1999), 385-391.

[6] Jolany, H, Mohebbi, H, Some results on generalized multi-poly Bernoulli and Euler polynomials, Int. J. Math. Comb., 2(2011), 117-129.

[7] Kaneko, M, Poly-Bernoulli numbers, J. de Theorie de Nombres 9 (1997), 221228.

[8] Khan W. A, A note on degenerate Hermite poly-Bernoulli numbers and polynomials, Journal of Classical Anal., 8(1)(2016), 65-76.

[9] Khan W. A, A new class of Hermite poly-Genocchi polynomials, J. Anal. Numb. Theor., 4(1)(2016), 1-8.

[10] Kim, T, Kwon, H. I, Lee, S. H and Seo, J. J, A note on poly-Bernoulli numbers and Polynomials of the second kind, Adv. Differen. Equations, 2014, 2014:219.

[11] Kim, D. S and Kim, T, A note on degenerate poly-Bernoulli numbers and polynomials, Advan. Diff. Equat., DOI 10.1186/s13662-015-0595-3, (2015).

[12] Kim, D. S, Dolgy, D. V and Komatsu, T, Barnes type degenerate Bernoulli polynomials, Adv. Stud. Contemp. Math., 25(1)(2015), 121-146.

[13] Kim, T, Barnes type multiple degenerate Bernoulli and Euler polynomials, Appl. Math. Comput., 258(2015), 556-564.

[14] Young, P. T, Degenerate Bernoulli polynomials generalized factorials sums and their application, J. Number Theory, 128(4)(2008), 738-758. 